\title{
L'intentionnalité cognitive et ses modes : Reinach critique de Brentano
}

\author{
Arnaud Dewalque
}

Je m'intéresserai ici à l'analyse descriptive des phénomènes judicatifs. La position d'Adolf Reinach, sur ce point, est profondément originale et particulièrement fine. D'une part, Reinach hérite de la conception thétique du jugement mise en avant par Franz Brentano, donc de l'idée que juger est un acte positionnel, thétique plutôt que synthétique. Mais d'autre part, il s'écarte fortement des versions de cette conception développées par Brentano (1874), Twardowski (1884), Windelband (1894) ou Husserl (1905) - pour ne citer que les plus célèbres ${ }^{1}$-, en « enrichissant » la théorie du jugement «au moyen d'une série de distinctions phénoménologiques» nouvelles et subtiles ${ }^{2}$. Il en résulte une théorie du jugement qui est certainement plus sophistiquée que celles de ses prédécesseurs et qui embrasse au passage une large variété d'états et d'actes « cognitifs » au sens le plus large: penser, questionner, représenter, connaître, croire, asserter, affirmer, nier, supposer, donner son assentiment, etc.

Dans les pages qui suivent, je suggérerai que la théorie reinachienne du jugement peut être vue, pour cette raison, comme une contribution à une théorie générale de l'intentionnalité cognitive et de ses modes. À cette fin, je focaliserai mon attention sur certaines divergences significatives entre Reinach et Brentano. Après quelques réflexions introductives ( $\S 1-2)$, j'examinerai plus exactement les questions suivantes : quels sont les motifs théoriques qui ont conduit Reinach à s'écarter de la théorie brentanienne du jugement (§§ 3-4) ? Et que retenir de ses analyses aujourd'hui (§§5-6) ?

\footnotetext{
${ }^{1}$ Cf. F. Brentano, Psychologie vom empirischen Standpunkte (= PES), Leipzig, Duncker \& Humblot, ${ }^{1} 1874$, ${ }^{2} 1911$ (rééd. dans Sämtliche veröffentlichte Schriften, Bd. 1, Frankfurt/Main, Ontos, 2008 ; trad. fr. M. de Gandillac, revue par J.-F. Courtine, Psychologie d'un point de vue empirique, Paris, Vrin, 2008); K Twardowski, Zur Lehre vom Inhalt und Gegenstand der Vorstellungen (1884), München-Wien, Philosophia, 1982 (trad. fr. J. English, « Sur la théorie du contenu et de l'objet des représentations », dans E. Husserl et K. Twardowski, Sur les objets intentionnels (1893-1901), Paris, Vrin, 1993, pp. 85-200); W. Windelband, Beiträge zur Lehre vom negativen Urteil (1884), éd. séparée, Tübingen, Mohr, ${ }^{2} 1921$ (trad. fr. É. Dufour, "Contributions à la théorie du jugement négatif», dans W. Windelband, Qu'est-ce que la philosophie ? et autres textes, Paris, Vrin, pp. 133-155); E. Husserl, Urteilstheorie. Vorlesung 1905, dans Husserliana Materialien (= HuaMat), Bd. V., Dordrecht, Kluwer, 2002.

${ }^{2}$ E. Husserl, "Adolf Reinach $\dagger »$, dans Kant-Studien 23 (1918), p. 148 ; repris dans Husserliana (= Hua), Bd. XXV : Aufsätze und Vorträge (1911-1921), Dordrecht, Nijhoff, 1987, p. 302.
} 
Comme on sait, Reinach appartient originairement au groupe des protophénoménologues ${ }^{3}$ munichois, constitué d'anciens étudiants de Theodor Lipps (18511914). Comme Johannes Daubert et d'autres, il a participé à ce que l'on appelle communément l' " invasion » de Göttingen par les Munichois, venus assister aux leçons de Husserl. Il a ainsi, en 1905, suivi le cours de Husserl sur la Théorie du jugement. Dans ce cours, Husserl soutient que son approche phénoménologique ne repose pas sur la "perception interne" (innere Wahrnehmung), mais sur la «perception des essences» (Wesenswahrnehmung). En ce sens, l'étude des structures fondamentales du psychique relève d'une discipline nouvelle, qui procède à des analyses d'essence (Wesensanalysen). «Cette discipline», écrit Husserl, «est celle que j'appelle phénoménologie; elle s'appellerait probablement encore mieux une théorie des essences (Wesenslehre) $»^{4}$.

Il est plausible que Reinach ait hérité directement de cette vision. Le phénoménologue ou (ainsi qu'il continue à l'appeler en 1914, contrairement à Husserl) le psychologue descriptif «n'a justement pas affaire à des faits, ne s'occupe ni d'expliquer des existences, ni de les reconduire à d'autres $»^{5}$. Pour cette raison, on a souvent de Reinach l'image d'un philosophe défendant une théorie «platonicienne » des essences, conçues comme des entités fixes et immuables. Quelle que soit la justesse de cette manière de voir, elle présente l'inconvénient de négliger un aspect important du programme phénoménologique de Reinach : l'essence d'un acte se manifeste toujours « en situation », à partir du sol vivant de l'expérience. Aussi Reinach met-il un soin tout particulier à traiter les phénomènes d'expression, notamment, à partir du contexte de l'énonciation. Ce constat explique bien sûr l'attention portée aux actes de langage et aux actes sociaux; mais il est également déterminant, selon moi, pour comprendre la conception reinachienne des actes judicatifs. Concrètement, Reinach soutient par exemple qu'un énoncé comportant une négation ne pourra pas être traité uniformément comme l'expression d'un jugement négatif ou d'un rejet, car il ne sera pas l'expression d'un même acte mental selon qu'il apparaît dans un contexte impliquant une prise de position personnelle ou dans un contexte narratif (par exemple, dans un livre d'histoire - j'y reviendrai). De surcroît, Reinach soutient qu'une théorie satisfaisante du jugement doit

\footnotetext{
${ }^{3}$ Sur l'existence d'une phénoménologie munichoise autonome, antérieure même au second tome des Recherches logiques de Husserl (1901), voir R. N. Smid, "Münchener Phänomenologie" - Zur Frühgeschichte des Begriffs », dans H. Spiegelberg et E. Avé-Lallemant (éds.), Pfänder-Studien, The Hague, Nijhoff, 1982, pp. 109-153, surtout pp. 115-116.

${ }^{4}$ E. Husserl, HuaMat V, pp. 36 et 38. Il ne faut sans doute pas surestimer l'importance de cette inflexion par rapport à Brentano, qui parle déjà d' « essence » (Wesen) dans ses cours de Phénoménologie descriptive, avec moins d'emphase.

${ }^{5}$ A. Reinach, «Über Phänomenologie » (1914), dans Sämtliche Werke (= SW), Bd. I, München, Philosophia, 1989, p. 534 (trad. fr. A. Dewalque, "Sur la phénoménologie », dans A. Reinach, Phénoménologie réaliste, Paris, Vrin, 2012, p. 39).
} 
tenir compte des "phénomènes d'accentuation» (Betönungsphänomene) ${ }^{6}$. Ces phénomènes, qui sont propres à ce qu'il nomme les «jugements polémiques » (e.g., «cette fleur n'est pas rouge», avec insistance sur la négation), constituent des modifications des actes judicatifs originaux par lesquelles ils cessent d'être les actes qu'ils étaient (une négation modifiée n'est plus une négation). Il n'apparaissent justement que lorsqu'on restitue le contexte des actes judicatifs.

Cette dimension " contextuelle » des analyses reinachiennes contribue à les rendre particulièrement fines et intéressantes. L'ambition de Reinach, dans ses travaux sur le jugement, est justement de décrire la nature ou l'essence des phénomènes judicatifs, avec toutes leurs nuances, tels qu'ils sont vécus dans l'expérience quotidienne.

Ses premiers travaux en ce sens remontent à l'été 1908. À cette époque, Reinach rédige un imposant manuscrit sur la théorie du jugement, qu'il défendra finalement comme thèse d'habilitation à l'Université de Göttingen au printemps 1909, sur la recommandation de Husserl. Pour une raison inconnue, le manuscrit de la thèse d'habilitation, intitulé Essence et systématique du jugement, ne sera jamais publié, contrairement à ce que Reinach annonce encore en 1911 (où il évoque une publication imminente sous le titre Jugement et état de choses), et il semble qu'aucune copie ne soit parvenue jusqu'à nous. Seul a été conservé un résumé, par Johannes Daubert, de l'un des rares exemplaires de la thèse d'habilitation qui étaient en circulation à l'époque ${ }^{7}$. Du rapport d'évaluation de Husserl, on peut toutefois établir que ce travail, d'au moins 277 pages, contenait d'imposants développements originaux, assortis d'une «critique polémique » qui était « principalement dirigée contre Brentano, Lipps » et Husserl luimême $^{8}$. L'article de Reinach sur le jugement négatif, paru en 1911 dans le Festschrift offert à Lipps, contient encore des traces de cette critique. Il constitue, avec le résumé de Daubert, la source principale dont nous disposons aujourd'hui pour reconstruire la théorie reinachienne du jugement.

\section{$2^{\circ}$ L'héritage ambivalent de Brentano}

Quels sont donc, d'après leurs caractéristiques "phénoménales », i.e. apparaissant dans l'expérience vécue, les actes ou états que l'on rassemble communément sous le titre de «jugement»? Qu'est-ce qui unit ces phénomènes judicatifs entre eux ? Et qu'est-ce qui les distingue d'autres actes ou états apparentés?

\footnotetext{
${ }^{6}$ Voir A. Reinach, «Wesen und Systematik des Urteils» (= WSU) (1908), SW I, p. 340 (trad. fr. P.-J. Renaudie, «Essence et systématique du jugement», dans A. Reinach, Phénoménologie réaliste, op. cit., p. 102).

${ }^{7}$ A. Reinach, « Wesen und Systematik des Urteils » (1908), SW I, pp. 339-346 (trad. fr., pp. 99-108).

${ }^{8} C f$. « Husserls Gutachten über Reinachs Habilitationsschrift », H. Spiegelberg éd., dans K. Mulligan (éd.), Speech Act and Sachverhalt. Reinach and the Foundations of Realist Phenomenology, Dordrecht, Nijhoff, 1987, pp. 253-256.
} 
Dans sa Psychologie d'un point de vue empirique (1874), Brentano a tâché de répondre à ces questions en établissant une « classification » des phénomènes psychiques qui soit conforme à leur nature. L'analyse descriptive, telle qu'elle est conçue par Brentano, consiste à dégager les «affinités naturelles »- et, pourrait-on ajouter, les contrastes naturels - qui structurent le domaine du mental ${ }^{9}$. Cette approche amène Brentano, d'abord, à identifier le caractère intentionnel comme critère privilégié du mental et, ensuite, à distinguer trois «classes fondamentales» (Grundklassen) correspondant à trois modes intentionnels irréductibles les uns aux autres : la classe des représentations, la classe des jugements et la classe des phénomènes affectifs (rassemblant les phénomènes d'amour/haine, d'attraction/répulsion). Brentano estime avoir démontré qu'il n'existe au fond que ces trois manières de se rapporter à un contenu, «ni plus ni moins ${ }^{10}$. Le sens dans lequel ces trois classes sont dites «fondamentales» n'est toutefois pas parfaitement clair. Elles sont, certes, impossibles à dériver les unes des autres $^{11}:$ j'aurai beau combiner des représentations, je n'obtiendrai jamais un jugement sans un acte spécifiquement judicatif, etc. Mais en affirmant le caractère fondamental de ces trois classes, Brentano semble supposer que tous nos phénomènes psychiques (perception, imagination, espoir, etc.) pourraient être reconstruits à partir d'elles à titre de phénomènes complexes.

C'est vraisemblablement cette dernière affirmation que prennent pour cible Husserl et Reinach. Aussi l'héritage brentanien est-il, à leurs yeux, profondément ambivalent. D’une part, ils reconnaissent à Brentano le mérite d'avoir conçu la multiplicité des états et des actes psychiques comme une multiplicité de modes intentionnels différents. D'autre part, ils lui reprochent d'avoir cherché à ramener la variété des modes intentionnels à un petit nombre de classes fondamentales. Leurs critiques se dirigent donc prioritairement contre la théorie brentanienne des "classes fondamentales", qu'ils interprètent - à tort ou à raison - dans un sens réductionniste.

Sur ce point, Husserl avait clairement annoncé sa position dès la cinquième Recherche logique, quoique de façon relativement modérée: "Quant à savoir si l'on considère comme pertinente la classification brentanienne des "phénomènes psychiques" [...], ce n'est pas de cela qu'il s'agit ici $»^{12}$. Tout ce qui importe dans un premier temps, poursuit-il, est de faire droit à la grande variété de modes intentionnels qui caractérisent des actes aussi différents que représenter, juger, supposer, douter, espérer, désirer, craindre, éprouver de la satisfaction ou du déplaisir, etc. Comment faire droit à la variété de ces modes intentionnels? Simplement en admettant l'impossibilité de «réduire»

\footnotetext{
${ }^{9}$ Voir, e.g., PES, ${ }^{2}$ 1911, Livre I, p. 62 (rééd. 2008, p. 59 ; trad. fr., p. 57).

${ }^{10}$ F. Brentano, PES, ${ }^{2} 1911$, Livre II, p. 124 (rééd. 2008, p. 285 ; trad. fr., p. 278).

${ }^{11}$ Ibid., ${ }^{2}$ 1911, Livre II, p. 125 (rééd. 2008, p. 286 ; trad. fr., p. 279).

${ }^{12}$ E. Husserl, Logische Untersuchungen, Bd. II/1 : Untersuchungen zur Phänomenologie und Theorie der Erkenntnis (LU I-V), Tübingen, Niemeyer, ${ }^{7} 1993, L U \mathrm{~V}, \S 10$ A 347/B 367 (= Hua XIX/1, p. 380 ; trad. fr. H. Élie, A. L. Kelkel et R. Schérer, Recherches logiques, t. II/1 et $2:$ Recherches sur la phénoménologie et la théorie de la connaissance, Paris, PUF, éd. revue, 1993 et 1996, pagination du texte all. en marge).
} 
(reduzieren) les «caractères intentionnels primitifs 》- Husserl précise dans la seconde édition (1913) : «d'après leur essence descriptive » - à d'autres genres de vécus ${ }^{13}$, bref : en adoptant une position anti-réductionniste.

De ce point de vue, la théorie brentanienne des classes fondamentales apparaît inévitablement comme suspecte. Ainsi, dans le cours de 1905 sur la théorie du jugement, Husserl insiste sur le fait que les théories de Brentano "sont dotées de grande valeur », mais « ne font pas droit à la multiplicité des phénomènes et $<$ aux $>$ concepts éidétiques (Wesensbegriffe) que l'on peut abstraire à partir d'eux $»^{14}$. Reinach, de son côté, s'oppose encore plus nettement à la tendance réductionniste qu'il diagnostique dans la psychologie brentanienne. Même si le nom de Brentano n'est pas explicitement mentionné, il fait clairement partie des auteurs ciblés par la critique virulente exposée dans la conférence de 1914 sur la phénoménologie. Vouloir «reconduire» (zurückführen) les expériences vécues à un nombre d'expériences fondamentales aussi petit que possible, en suivant la manière de procéder des sciences de la nature, c'est là, écrit Reinach, une tâche «dépourvue de sens » (sinnlos $)^{15}$. La classification brentanienne, sur ce point, ne serait finalement pas différente de celle de Kant, qui cherchait à ramener la vie mentale aux facultés de sentir, vouloir et penser. De telles «tentatives de reconduction», écrit Reinach, ne sont rien d'autre qu' " un appauvrissement et une falsification de la conscience », car elles conduisent à interpréter le vécu en le transformant en ce qu'il n'est pas, à effectuer une Umdeutung, une interprétation déformante ${ }^{16}$.

Dans sa théorie du jugement, Reinach entreprend précisément de faire droit à la variété des phénomènes liés, de près ou de loin, au jugement. Cette approche le conduit à rejeter la conception brentanienne des actes judicatifs sur plusieurs points tout à fait centraux. Il s'attaque, en particulier ${ }^{17}$, aux deux thèses brentaniennes suivantes :

(i) Juger est un phénomène bipolaire consistant à reconnaître comme vrai ou à rejeter comme faux (thèse de la bipolarité).

(ii) Tout jugement est nécessairement fondé sur une représentation (thèse de la fondation).

${ }^{13}$ Ibid., A 348/B 368 (= Hua XIX/1, p. 381).

${ }^{14}$ HuaMat V, p. 4-5. Husserl, qui évoque dans ce passage le «radicalisme de Brentano », ajoute : «Les choses ne sont malheureusement pas si simples que se le représentait Brentano ».

${ }^{15}$ A. Reinach, « Über Phänomenologie » (1914), SW I, p. 534 (trad. fr., p. 39).

${ }^{16} \mathrm{Id}$.

${ }^{17} \mathrm{Je}$ fais ici abstraction des attaques dirigées contre une troisième thèse brentanienne, à savoir la thèse de la simplicité : il existe des contenus représentationnels jugeables simples. Cf. PES, ${ }^{2} 1911$, Livre II, p. 49 (rééd. 2008 , p. 230 ; trad. fr., p. 228). Cette thèse concerne le contenu jugeable, c'est-à-dire le versant «noématique » des actes judicatifs, et non ces actes eux-mêmes. Reinach la rejette au profit de sa théorie des « états-de-choses ». Tout comme Frege (Begriffsschrift, Halle, Nebert, 1879, § 2 ; rééd. Hildesheim, Olms, 2007 , p. 2), Reinach soutient qu'un jugement ne peut jamais se diriger vers un objet, mais seulement vers un état-de-choses. Voir « Zur Theorie des negativen Urteils » (1911), SW I, p. 111 (trad. fr. M. de Launay revue par J.-F. Courtine, «La théorie du jugement négatif», dans A. Reinach, Phénoménologie réaliste, op. cit., p. 142). 
Considérons d'abord la thèse de la bipolarité judicative. Juger, écrit Brentano, ne consiste pas à relier des représentations entre elles, mais à « reconnaître » (Annehmen) ou à « rejeter» (Verwerfen) quelque chose comme étant, respectivement, vrai ou faux ${ }^{18}$. L'affirmation et la négation constitueraient donc ce que l'on pourrait appeler l'essence descriptive des actes judicatifs.

Cette thèse, dans le chef de Brentano, doit répondre à un double objectif : il s'agit, d'une part, de saisir ce qui unit l'ensemble des phénomènes judicatifs d'après leur « nature » et, d'autre part, de montrer ce qui les distingue de tous les autres phénomènes psychiques. Pour atteindre cet objectif, il faut donc que la thèse de la bipolarité judicative (reconnaissance-rejet) satisfasse deux conditions : elle est censée rendre compte de tous les phénomènes judicatifs et seulement des phénomènes judicatifs. Or, selon Reinach, ces deux conditions ne sont pas remplies. La thèse brentanienne de la bipolarité ne permet ni d'appréhender l'unité fondamentale de la classe des jugements, ni de délimiter adéquatement cette classe face aux phénomènes non judicatifs, et ce pour trois raisons au moins.

$1 /$ D'abord, il existe quantité de cas où la présence effective d'une négation n'implique aucun acte de rejet ni même, plus largement, aucun acte de jugement. La thèse de Brentano a, en effet, une conséquence difficilement tenable : elle implique qu' «il suffit de définir quelque chose comme mettant en œuvre une négation pour le qualifier aussitôt de jugement ${ }^{19}$. Or, Reinach soutient que cette implication est fausse. Considérons les énoncés suivants : $<A$ n'est pas $b$, je le crains bien $>,<A$ n'est-il pas $b$ ? $>$, $<$ À supposer que $A$ ne soit pas $b>$, etc. Dans tous ces cas, il y a une dénégation (un $n e \ldots$ pas), mais il n'y a pas de négation à proprement parler ni, par conséquent, de rejet. La présence d'une négation ne peut donc servir de critère pour délimiter la sphère des actes de rejet - ni, à plus forte raison, la sphère des actes judicatifs.

2 / Pour fonder l'unité de la classe des actes judicatifs, il faut que le critère soit parfaitement univoque - sans quoi on n'obtiendrait qu'une unité d'homonymie (différents phénomènes peuvent être désignés du même nom), mais non une véritable unité descriptive. Or, une importante objection avancée par Reinach concerne l'équivocité des termes Anerkennen-Verwerfen. La terminologie de la reconnaissance et du rejet véhicule effectivement un certain nombre d'ambigüités. D'abord (a), ces termes peuvent avoir une connotation axiologique : reconnaître serait attribuer une valeur en fonction d'un but ; juger, ce serait « évaluer». Brentano et Marty, estime Reinach, ont eu raison d'écarter cette variante de la conception thétique, introduite exemplairement par Windelband ${ }^{20}$.

\footnotetext{
${ }^{18}$ PES, ${ }^{2} 1911$, Livre II, p. 34 (rééd. 2008, p. 219 ; trad. fr., p. 217).

${ }^{19}$ " Zur Theorie des negativen Urteils» (1911), $S W$ I, p. 131 (trad. fr., p. 165).

${ }^{20}$ Voir A. Dewalque, "Windelband on Beurteilung », dans M. Van der Schaar (éd.), Judgement and the Epistemic Foundation of Logic, Dordrecth, Springer, 2013, pp. 85-89.
} 
Ensuite (b), la terminologie brentanienne peut engendrer une confusion avec l'assentiment (Zustimmung). Selon Reinach, lorsque je donne mon assentiment à une assertion qui m'est présentée, et que je juge : <oui, A est effectivement b>, les mots $<$ oui $>$ et $<\mathrm{A}$ est effectivement $\mathrm{b}>$ expriment deux genres de « reconnaissance » tout à fait différents. $<A$ est effectivement $b>$ est l'expression d'un acte judicatif dirigé vers l'être-b de A, mais <oui> est l'expression d'un acte d'assentiment dirigé, non pas vers l'être-b de A, mais vers l'acte judicatif lui-même. C'est donc un acte de second degré. L'idée de Reinach est que l'on ne peut donner son assentiment (ou le refuser) qu'à un acte de jugement, non à un état de choses (l'être-b de A). Le terme Anerkennung pouvant désigner aussi bien l'assentiment que l'acte judicatif, il ne permet pas de délimiter les phénomènes judicatifs stricto sensu. Ici encore, la théorie de la reconnaissance et du rejet, pour ainsi dire, embrasse trop large : certes, elle capture tous les jugements, mais elle ne capture pas seulement les jugements.

3 / En outre, il paraît tout à fait plausible de soutenir qu'il y a une communauté entre affirmation et négation - communauté à laquelle l'interrogation, par exemple, ne participe pas. Une théorie du jugement satisfaisante doit saisir leur «affinité, du point de vue descriptif $»^{21}$. Or, la théorie brentanienne ne permet pas de comprendre ce qui unit les actes de reconnaissance et de rejet. Il faut pourtant qu'ils présentent un point commun ou, plutôt, une «partie abstraite», un «aspect» ou un «moment» commun, si reconnaissance et rejet peuvent bien être traités comme des qualités judicatives, comme des manières de juger. Chez Brentano, l'unité des deux qualités judicatives ne peut reposer, en définitive, que sur le concept de belief qui est emprunté à l'empirisme de Hume et de Mill. Malheureusement, à suivre Reinach, cette issue n'est pas satisfaisante, car le concept de jugement est plus large que le concept de croyance. Certes, d'un côté, juger est un acte de croyance ou une conviction (disons, pour faire bref, un acte doxique); mais d'un autre côté, juger est aussi asserter (Behaupten). Reinach en appelle ici au langage ordinaire. Lorsqu'on parle de "porter un jugement», on ne veut pas dire endosser une croyance, mais réaliser une assertion. Le caractère assertif est commun aux affirmations et aux négations. Considérons l'énoncé suivant ${ }^{22}:<$ Le roi n'était pas énergique>. Cet énoncé peut exprimer une prise de position de l'historien dans un contexte polémique, donc un acte de rejet dirigé contre l'affirmation contraire. Mais s'il s'agit d'un simple élément narratif, qui prend place dans la description historique $(<\mathrm{A}$ cette époque, le pays redevint florissant. Le roi n'était pas précisément énergique, mais...>), dans ce cas, la négation n'est plus l'expression d'un rejet, mais il est «bien plus évident», écrit Reinach, de traiter la phrase entière comme l'expression d'une position ou d'une assertion. Ainsi, «c'est seulement l'aspect assertif (Behauptungsmoment) qui fait des vécus de pensée, avec ou sans négation, des

${ }^{21}$ «Zur Theorie des negativen Urteils » (1911), SW I, p. 127 (trad. fr., p. 161).

22 «Wesen und Systematik des Urteils » (1908), SW I, p. 340 (trad. fr., p. 101). 
jugements. On ne doit parler d'affirmation et de négation que si l'on comprend par négation une assertion à l'intérieur de laquelle on nie $»^{23}$. Un jugement affirmatif se définit alors simplement de façon privative : une affirmation, c'est une assertion au sein de laquelle n'intervient pas la « fonction de dénégation ».

Reste alors à répondre à la question suivante : qu'est-ce qui justifie l'introduction de la distinction, absente chez Brentano, entre conviction et assertion ? Reinach avance trois critères descriptifs :

1 / La conviction admet des degrés, l'assertion non. L'idée d'une gradualité de l'assertion ou d'un acte plus ou moins assertif est tout simplement un non-sens : « Soit quelque chose est asserté, soit il ne l'est pas. Mais il n'y a pas de degrés dans l'assertion $»^{24}$. En revanche, la conviction peut décroître progressivement jusqu'au doute en passant par la présomption : il y a bien un sens à traiter la croyance ferme qu'il pleut en ce moment sur Paris, la supposition qu'il pleut en ce moment sur Paris, et le doute qu'il pleut en ce moment sur Paris, comme une série unitaire d'actes doxiques dans lesquels la conviction se modifie graduellement en doute.

2 / De façon très remarquable, la distinction reinachienne entre conviction et assertion peut être considérée comme un cas particulier de la distinction plus générale entre état mental et acte ou événement mental. La conviction, tout comme la présomption et le doute, est un état mental; à ce titre, elle possède une certaine durée ou une certaine épaisseur temporelle. L'assertion, par contraste, est un acte ponctuel qui a lieu à l'instant $t$, qui peut certes être répété à l'instant $t+1$, mais qui - par essence - ne peut pas durer ou avoir des «parties temporelles». Ainsi, cela a un sens de dire que, depuis plusieurs heures, j'ai la conviction qu'il pleut sur Paris. Mais cela n'a pas de sens de dire que, durant tout ce temps, j'étais en train d'affirmer qu'il pleuvait sur Paris : «[Une] conviction peut durer un temps quelconque, [une] assertion [par contre] n'est pas un caractère de l'état du Je [Zuständlichkeit des Ich] [et] ne peut pas durer ${ }^{25}$.

3 / Enfin, il y a, entre conviction et assertion, un rapport de «séparabilité unilatérale », au sens que Brentano donne à cette expression dans ses cours de psychologie descriptive $^{26}$. L'assertion - il faudrait dire : l'assertion véritable et sincère, qui exclut le mensonge - présuppose une conviction : « Aucune assertion n'est possible si elle n'est pas accompagnée d'une conviction à son fondement »- conviction qui doit être, cela va de soi, de même contenu que l'assertion qui s'édifie sur elle ${ }^{27}$. Autrement dit, «toute assertion repose sur une conviction $»^{28}$, l'inverse n'étant pas vrai. Je peux être convaincu qu'il pleut en ce moment sur Paris sans nécessairement affirmer qu'il pleut en

\footnotetext{
${ }^{23} I d$. (trad. fr. modifiée, p. 102). Cette position est évidemment très proche de celle de Frege.

${ }^{24}$ «Zur Theorie des negativen Urteils» (1911), SW I, p. 99 (trad. fr. modifiée, p. 128).

${ }^{25}$ « Einleitung in die Philosophie » (1913), SW 1, p. 425.

${ }^{26}$ Voir F. Brentano, Deskriptive Psychologie, Hamburg, Meiner, 1982, pp. 12-13.

27 « Zur Theorie des negativen Urteils » (1911), SW I, p. 100 (trad. fr., p. 129).

${ }^{28}$ Ibid., p. 125 (trad. fr. modifiée, p. 159).
} 
ce moment sur Paris. Bref: «La conviction peut exister sans assertion, mais non l'inverse $»^{29}$.

Cette analyse (ou quasi-analyse) ${ }^{30}$ des phénomènes judicatifs débouche sur une profonde réforme de la conception thétique ou positionnelle. Fondamentalement, le caractère thétique est pris en charge par l'assertion : "Juger, c'est asserter » (Urteilen ist Behaupten $)^{31}$. Une conviction isolée ne sera pas encore traitée comme un jugement stricto sensu. Sur ce point, l'analyse développée plus tard par un autre philosophe issu du cercle munichois, Alexander Pfänder, rejoint directement celle de Reinach. Comme Reinach, Pfänder, dans sa Logique (1921), isole justement l' «aspect assertif» (Behauptungsmoment) comme l' " élément qui seul caractérise proprement le jugement en tant que tel ». L'assertion n'est pas une composant des actes judicatifs parmi d'autres, mais elle est l' " essence caractéristique du jugement 》 (das charakteristische Wesen des

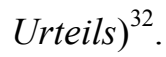

\section{$4^{\circ}$ Représenter et vouloir-dire}

Les observations qui précèdent ne constituent qu'une partie de la réforme de la théorie du jugement entreprise par Reinach. Comme je l'ai annoncé, ce dernier s'attaque également à la deuxième thèse de Brentano :

(ii) Tout jugement est fondé sur une représentation.

Avant toute chose, il ressort de la section précédente que le mot «jugement» est ici ambigu, puisqu'il peut être entendu au sens de «conviction » ou au sens d' "assertion ». Il convient donc de distinguer deux variantes de cette thèse :

(ii-a) Toute conviction est fondée sur une représentation

(ii-b) Toute assertion est fondée sur une représentation

Cela étant posé, Reinach considère, à la suite de Husserl, que le terme « représentation » est équivoque. L'innovation principale de Reinach, sur ce point, consiste à introduire une nouvelle distinction entre représenter et penser, viser ou vouloir-dire (Meinen). Au sens où la conçoit Reinach, cette nouvelle distinction ne coïncide pas avec intuition et pensée. Même si le cas est plus rare, il peut y avoir des représentations non intuitives, de même qu'il peut y avoir des pensées qui sont, d'une manière ou d'une autre, accompagnées d'intuition.

${ }^{29}$ A. Reinach, « Einleitung in die Philosophie » (1913), SW 1, p. 425.

${ }^{30}$ Sur ce terme, $c f$. D. Seron, Ce que voir veut dire, Paris, Le Cerf, 2012, pp. 166-167.

${ }^{31}$ A. Reinach, «Wesen und Systematik des Urteils » (1908), SW I, p. 341 (trad. fr. modifiée, p. 103).

${ }^{32}$ A. Pfänder, Logik, M. Crespo éd., Heidelberg, C. Winter, ${ }^{4} 2000$, pp. 40-41. 
Pour clarifier cette distinction, Reinach propose l'exemple suivant. Lorsque quelqu'un énumère rapidement les noms des montagnes d'Allemagne, il y a une différence phénoménologique entre énumérer les noms en comprenant ce qui est dit et énumérer les noms sans comprendre de quoi il est question. L'énonciation accompagnée de compréhension est fondée sur un type d'acte particulier que Reinach appelle le Meinen (penser, vouloir-dire). Les actes de Meinen se caractérisent par le fait de «viser» (abzielen auf) quelque chose. Ces actes forment le soubassement spontané ou « actif» de notre vie cognitive. À ce titre, ils doivent être soigneusement distingués des actes de représentation, qui sont en quelque sorte leur pendant «passif». Être-représenté est synonyme d'être-là (Da-Sein); viser, penser ou vouloir dire quelque chose, c'est se rapporter à quelque chose qui n'est pas là pour nous, à quelque chose de non présent ${ }^{33}$. Reinach invoque, à nouveau, trois critères descriptifs-eidétiques pour fonder cette distinction.

1 / Le premier critère est un critère de séparabilité mutuelle ou bilatérale au sens de Brentano: on peut viser sans représenter, et inversement. Bien plus, les actes de représentation et de pensée ne sont pas seulement séparables; ils s'excluent mutuellement. Ainsi, quand je vois un livre, je ne pense pas à sa face arrière. Je ne l'imagine pas non plus ${ }^{34}$. Mais je me la représente ou j'en ai une représentation, à savoir justement une représentation non intuitive ou, disons, relativement non intuitive : la face arrière est co-représentée en même temps que la face visible (qui est la seule à être représentée intuitivement au sens propre du terme). Pour employer les termes de Reinach lui-même : «Quand je représente l'objet, je ne le pense plus » ${ }^{35}$. Inversement, je n'ai pas besoin de me représenter un objet pour le viser de façon intellective.

2 / Ensuite, Reinach soutient que la pensée s'accomplit nécessairement dans le langage: viser, c'est toujours viser à travers des mots. La visée est «habillée linguistiquement » (sprachlich eingekleidet $)^{36}$. Les actes de représentation, quant à eux, ne seraient pas intrinsèquement liés au langage.

3 / Enfin, les actes de représentation sont pluri-modaux : ils se déclinent selon des modalités différentes en fonction de ce qui est représenté ${ }^{37}$. Avoir la représentation d'une couleur implique un acte de vision; avoir la représentation d'un son implique un acte d'audition; avoir la représentation d'un nombre signifie le penser, l'appréhender intellectuellement; avoir la représentation d'une valeur implique qu'on la ressente, etc. Tous ces actes, pris en eux-mêmes, sont intrinsèquement différents. Ils constituent autant de manières d'être-là ou d'être-présent pour nous. La diversité des contenus représentés

\footnotetext{
${ }^{33}$ Le concept de Meinen était précisément employé en ce sens par Pfänder dans sa Phénoménologie des Wollens. Eine psychologische Analyse, Leipzig, Barth, 1900, pp. 22 sq., dont Reinach s'est vraisemblablement inspiré.

${ }^{34}$ A. Reinach, «Zur Theorie des negativen Urteils » (1911), SW I, p. 105 (trad. fr., p. 135).

${ }^{35}$ A. Reinach, «Wesen und Systematik des Urteils » (1908), SW I, p. 339 (trad. fr. modifiée, p. 100).

${ }^{36}$ A. Reinach, «Zur Theorie des negativen Urteils » (1911), SW I, p. 102 (trad. fr. modifiée, p. 132)

${ }^{37}$ Ibid., SW I, p. 103-104 (trad. fr., p. 133-134).
} 
implique diverses manières de représenter ou divers modes de représentation. La pensée, en revanche, est uni-modale. Que je parle de la couleur rouge, du son do, du nombre trois ou de quoi que ce soit d'autre, dans tous les cas, si je comprends les mots que j'emploie, je me rapporte de façon uniforme à ce qui est visé. Il y a certes, dans ce cas, des différences de contenu ou d'objectualité visée, mais ces différences ne correspondent pas à des différences d'actes. La diversité des contenus pensés n'implique pas diverses manières de penser.

Revenons à la thèse de la fondation. Cette thèse s'est dédoublée sur le versant doxique et sur le versant assertif. Comme le remarque Reinach, la thèse (ii-b) est fausse, si l'on entend par là qu'une assertion devrait nécessairement tirer son caractère directionnel d'actes de représentation sous-jacents. Nous pouvons asserter quantité de choses (par exemple, que $<$ Paris est la capitale de la France $>$ ou que $<$ la racine carrée de trois est un nombre réel $>$ ) sans en avoir une représentation au sens strict. En revanche, il est vrai que l'assertion doit tirer sa directionnalité de quelque part, et que seuls des actes spontanés de visée peuvent assurer cette fonction objectivante. Le Meinen constitue un «soubassement nécessaire » du Behaupten ${ }^{38}$. Il y a donc nécessairement, dans tout acte judicatif total, une "composante de visée » (Meinensbestandteil) en vertu de laquelle l'acte est dirigé vers tel ou tel état de choses. Cette composant peut naturellement être commune à une question et à un jugement. Lorsque je soulève la question $<$ pleut-il en ce moment sur Paris ?>, je vise le même état de choses que lorsque j'affirme qu' $<$ il pleut en ce moment sur Paris $>$. Le Meinen est donc un élément nécessaire mais non suffisant pour accomplir un acte interrogatif ou assertif.

Reste à évaluer la thèse (ii-a), selon laquelle toute conviction repose nécessairement sur une représentation ou implique une représentation à son fondement. Il est vrai, remarque Reinach, qu'un état doxique peut être directement motivé par un acte de représentation. Supposons que vous vous trouviez devant une fleur et que vous perceviez sa couleur rouge. Sur la base de cette expérience, vous êtes fermement convaincu que la fleur est rouge. Votre conviction est fondée sur la représentation (visuelle) de la fleur en tant que rouge; elle est une conviction que la fleur est rouge, précisément en vertu de la représentation qui lui confère sa directionnalité particulière. Ce constat semble plaider en faveur de la thèse brentanienne. Il semble toutefois nécessaire d'y ajouter une importante restriction. La fondation des états doxiques sur des représentations, précise Reinach, est possible mais non nécessaire. Dans l'exemple mentionné, il est en effet parfaitement concevable que vous restiez convaincu que la fleur est rouge alors même que vous n'en avez plus aucune représentation effective: la conviction peut perdurer alors que la représentation s'est éteinte ${ }^{39}$. Conformément à cela, il semble que le rapport entre conviction et représentation soit un rapport de séparabilité bilatérale ou mutuelle au sens

${ }^{38}$ A. Reinach, « Zur Theorie des negativen Urteils » (1911), SW I, p. 107 (trad. fr. modifiée, pp. 137-138).

${ }^{39}$ Ibid., p. 108 (trad. fr., p. 138). 
de Brentano - ce qui exclut un rapport de fondation au sens strict (lequel correspond à un rapport de séparabilité unilatérale: A ne peut exister sans B).

\section{$5^{\circ}$ Variétés d'intentionnalité cognitive}

Ce bref aperçu suffit à montrer que les analyses de Reinach débordent de loin le cadre strict de la théorie du jugement. Elles embrassent en fait une large variété d'états et d'actes intervenant dans les processus cognitifs au sens le plus large (que l'on opposera aux phénomènes émotionnels). Ce constat suggère que la théorie reinachienne $d u$ jugement peut être comprise comme une contribution à un programme descriptif plus vaste, dont la clarification des actes judicatifs stricto sensu ne constitue qu'une portion. C'est manifestement en ce sens que Husserl lui-même envisageait sa propre théorie des actes judicatifs dans son cours de 1905 : «Il n’y a pas de théorie phénoménologique du jugement par soi, il y a seulement une analyse eidétique englobante des actes objectivants et, à l'intérieur de celle-ci, une analyse du jugement qui s'y trouve co-incluse (mitbeschlossen) $»^{40}$. À mon sens, c'est précisément sur ce terrain, à savoir celui d'une étude de l'intentionnalité cognitive et de ses modes, que les résultats des analyses de Reinach peuvent être réactualisés aujourd'hui.

Considérons l'exemple célèbre avancé par Galen Strawson en 1994 et régulièrement invoqué par les partisans d'une "phénoménologie cognitive». Strawson imagine un scénario mettant en scène deux individus, un anglophone ignorant le français (Jack) et un francophone (Jacques), qui écoutent les nouvelles en français. Il semble plausible d'affirmer que ces deux individus ont des expériences différentes, alors même qu'ils entendent strictement la même séquence verbale, donc qu'ils ont la même expérience auditive $^{41}$. À la rigueur, on peut objecter que leur expérience auditive diffère en ceci que Jacques segmente automatiquement le flux sonore en mots, alors que cette opération ne se produit pas - ou, du moins, pas de la même manière - chez Jack. Mais cette différence est négligeable, soutient Strawson, car il est possible d'imaginer un cas plausible où les locuteurs, parlant la même langue, ont une expérience auditive identique (segmentent le flux auditif de la même manière), mais où l'un comprend ce qu'il entend et l'autre non (à supposer, par exemple, qu'il s'agisse d'un message codé dont l'un des deux locuteurs seulement détient la clé) ${ }^{42}$. Bref, l'idée de Strawson est simplement la suivante : alors même que l'expérience perceptuelle est identique, un locuteur peut comprendre les sons entendus, les appréhender immédiatement comme des signes faisant référence à des phrases, et l'autre non.

\footnotetext{
${ }^{40}$ E. Husserl, HuaMat V, p. 101.

${ }^{41}$ G. Strawson, Mental Reality, Cambridge MA, MIT Press, 1994, p. 5-6.

${ }^{42}$ Ibid., p. 6.
} 
Indépendamment des conclusions (anti-intentionnalistes) que Strawson pense devoir tirer de ce scénario, on se posera les deux questions suivantes. D'abord (a), comment décrire cette expérience cognitive, au demeurant tout à fait courante? Quels sont les aspects constitutifs de l'expérience consistant à entendre des mots en les comprenant ? Ensuite (b), qu'est-ce qui distingue la connaissance proprement dite de tous les phénomènes apparentés ou concomitants (croyance, jugement, etc.) ?

1 / Dans le cadre de l'esquisse fournie par Brentano, l'audition avec compréhension relève de la sphère générale des représentations. C'est là une conséquence directe de son concept large de représentation et de l'idée que seules les représentations peuvent être objectivantes ou fournir une direction intentionnelle : «Lorsque j'entends et comprends un nom, je me représente ce qu'il désigne; et les noms n'ont en général d'autre but (Zweck) que de provoquer des représentations (Vorstellungen hervorzurufen) ${ }^{43}$. Les analyses de Reinach ont toutefois introduit une importante prise de distance à l'égard d'une telle conception. Contre la tendance réductionniste à uniformiser le soubassement des actes cognitifs dans un concept unitaire de « représentation », Reinach privilégie une approche différenciée, sensible à la distinction entre représenter et viser ou vouloir-dire. De manière intéressante, le caractère réceptif de l'expérience de compréhension auditive, telle qu'elle est illustrée dans l'exemple de Strawson, exclut toutefois qu'on la caractérise comme un acte de visée ou de vouloir-dire. La raison en est que l'intentionnalité du Meinen est, comme dit Reinach, une «intentionnalité spontanée » ${ }^{44}$. L'intentionnalité de la compréhension auditive, par contre, est une intentionnalité réceptive (on reconnait l'emprunt du couple spontanéité-réceptivité au kantisme). Mais par ailleurs, elle ne peut pas non plus être décrite comme une représentation, car conformément aux développements précédents, on ne peut parler de représentation au sens strict que lorsque l'objet ou l'état de choses « est là », lorsqu'il est directement présent à notre esprit ${ }^{45}$. Or, il est clair que, dans l'exemple de Strawson, Jacques peut comprendre les phrases qu'il entend sans pour autant se représenter ce dont il est question. Il semble donc que le phénomène de compréhension auditive ne relève ni du Meinen, ni du Vorstellen, mais d'un autre mode d'intentionnalité, encore à définir, qui serait quelque chose comme une «visée passive».

2 / Qu'est-ce que connaître stricto sensu ? Quelle est l'essence descriptive des actes cognitifs ? Sur ce point aussi, les analyses de Reinach sont particulièrement fines et intéressantes. Elles se résument grosso modo à l'idée suivante. Classiquement, la connaissance est assimilée au jugement. Connaître, c'est juger. Cependant, estime Reinach, « il nous faut distinguer de la manière la plus nette jugement et connaissance » (contre Meinong, notamment ${ }^{46}$. Tous les jugements ne sont pas des connaissances. Un

${ }^{43}$ F. Brentano, PES, ${ }^{2} 1911$, Livre II, p. 34 (rééd. 2008, p. 219 ; trad. fr., p. 217).

${ }^{44}$ " Zur Theorie des negativen Urteils » (1911), $S W$ I, pp. 102 et 108 n. (trad. fr., pp. 131 et 138 n.).

${ }^{45}$ Ibid., p. 108 n. (trad. fr., p. 138 n.).

${ }^{46}$ Ibid., p. 120 (trad. fr., p. 152). 
étudiant en mathématiques peut croire sur parole ce que lui enseigne sont professeur : il peut penser que la somme des angles d'un triangle est égale à deux droits, être convaincu de la justesse de cette affirmation et former un acte assertif correspondant. Peut-on, dans ce cas, parler de connaissance ? Reinach répond négativement : une assertion que nous effectuons en nous en remettant à une autorité extérieure n'est pas une connaissance. On ne peut parler de connaissance qu'à partir du moment où l'élève dispose d'une vision claire (Einsicht) et évidente. Les actes assertifs, considérés en eux-mêmes, sont «aveugles» (blind), non évidents ${ }^{47}$. De ce point de vue, Reinach suggère que «la conviction est probablement plus importante pour le jugement, car elle seule voit et est à proprement parler connaissance $»^{48}$.

Cela étant dit, une analyse descriptive plus fine peut mettre au jour des différences descriptives fondamentales entre la connaissance et la conviction. Tout d'abord (a), on procédera ici comme partout ailleurs, à savoir en étudiant les relations de séparabilité (unilatérale ou mutuelle) ou d'inséparabilité (unilatérale ou mutuelle) entre les phénomènes psychiques. De ce point de vue, il semble évident qu'il peut y avoir une conviction sans connaissance - par exemple, je peux me souvenir que la fleur que j'ai vue est rouge, et en être fermement convaincu. La conviction étant un état (et non un acte), elle peut perdurer alors même que je n'ai plus une vision claire et évidente (i.e., une connaissance) de la rose comme étant rouge, mais seulement un souvenir plus ou moins fiable. Ensuite (b), la connaissance, comme l'assertion, n'est pas graduelle : soit je sais que la rose est rouge, soit je ne le sais pas (mais le présume, le devine, en ai un vague souvenir, etc.). Enfin (c), la connaissance, comme l'assertion également, est ponctuelle ; à l'inverse, la conviction est un état, elle a une durée. C'est pourquoi on peut dire que la conviction "dépasse en durée la connaissance» et qu'elle "peut même se prolonger alors que l'état de choses n'est plus présent $»{ }^{49}$.

Partant, la connaissance doit être conçue comme un acte sui generis, distinct des actes apparentés de compréhension auditive, de vouloir-dire, de présentification (souvenir), de conviction et d'assertion. Tous ces actes ou états jouent un rôle dans nos processus cognitifs et peuvent y intervenir à titre de composantes ou de parties abstraites. Il reste que la connaissance elle-même ne se réduit à aucun de ces phénomènes : «Connaître, ce n'est pas présentifier, mais ce n'est pas non plus évidemment affirmer un état de choses. Ce qui est, en effet, essentiel à la connaissance, c'est qu'en elle l'état de choses corrélatif soit là pour nous au sens prégnant du terme; dans l'affirmation, au contraire, l'état de choses est simplement visé $»^{50}$.

\footnotetext{
${ }^{47}$ Ibid., p. 119 (trad. fr., p. 151). Sur ce point précis, Reinach rejoint Brentano.

48 "Wesen und Systematik des Urteils » (1908), SW I, p. 343 (trad. fr., modifiée, p. 106).

49 «Zur Theorie des negativen Urteils » (1911), SW I, p. 125 (trad. fr., p. 158).

${ }^{50}$ Ibid., p. 119 (trad. fr., p. 152).
} 
La théorie reinachienne du jugement, on l'a vu, constitue un profond remaniement de l'héritage brentanien. Ce remaniement, me semble-til, présente au moins deux avantages.

1 / Contre la thèse de la bipolarité judicative, Reinach soutient que tout acte judicatif au sens plein est un acte assertif (assertion) fondé sur un état doxique (conviction). Cette manière de voir a l'avantage de résoudre un certain nombre de difficultés qui semblent malaisées à surmonter dans le cadre théorique brentanien. D'abord (a), elle fournit un critère clair permettant d'indiquer à partir de quel moment on entre dans la sphère du jugement. Elle permet simultanément de comprendre en vertu de quoi un acte de représentation ou d'interrogation, par exemple, se distinguent de l'acte de jugement correspondant (i.e. de même contenu). Ensuite (b), la distinction entre conviction et assertion permet de résoudre le problème du caractère graduel ou non $\mathrm{du}$ jugement, en suggérant que la gradualité ne s'applique qu'à la composante doxique de la théorie du jugement, donc à la conviction, mais non à l'assertion (non graduelle).

L'analyse de Reinach, sur ce point précis, apparaît d'autant plus précieuse que la question de la gradualité des actes judicatifs avait fait l'objet de vifs débats dans les versions antérieures de la conception thétique forgées par Brentano et Windelband ${ }^{51}$. Or, aussitôt que l'on sépare avec Reinach la conviction de l'assertion, la controverse sur la gradualité se ramène à un faux problème. L'erreur commune de Brentano et de Windelband, si l'on peut dire, était de considérer le terme « jugement » comme univoque, l'un soutenant que le jugement est un phénomène admettant de degrés intermédiaires entre affirmation et négation (Windelband), l'autre refusant catégoriquement l'idée d'une gradualité judicative (Brentano). La théorie de Reinach permet de connaître aux deux positions un noyau descriptif correct: lorsque Windelband introduit la notion d' " évaluation» graduelle, c'est en fait le concept de conviction qui est visé : une conviction peut être, en effet, plus ou moins forte. À l'inverse, lorsque Brentano soutient qu'un acte judicatif n'admet pas de plus et de moins (car on ne peut reconnaître quelque chose comme « plus ou moins vrai » ni le rejeter comme «plus au moins faux »), c'est en fait la notion d'assertion qui est visée.

2 / Un autre mérite de la théorie de Reinach est d'avoir exploré à nouveaux frais les soubassements des actes de conviction et d'assertion. Sa principale innovation, sur ce point, consiste à avoir fait une place au concept de «visée» ou de «vouloir-dire» (Meinen), par opposition au concept étroit de « représentation ». J'ai suggéré, dans le $\S 5$, que ses recherches offrent ainsi un cadre théorique particulièrement fructueux pour forger quelque chose comme une analyse des phénomènes psychiques cognitifs. Dans cette perspective, il n’y a toutefois pas lieu de développer une «phénoménologie cognitive »

${ }^{51}$ Voir A. Dewalque, « Windelband on Beurteilung », art. cit., § 5. 
qui serait distincte (et indépendante) de l'analyse descriptive des actes et états intentionnels. La phénoménologie des actes et états cognitifs n'est précisément rien d'autre que l'analyse des modes intentionnels qui constituent la vie cognitive dans toute sa richesse, et qui apparaissent ou se donnent phénoménalement dans l'expérience vécue. 